\title{
Impaired expression of genes coding for reactive oxygen species scavenging enzymes in testes of Mtfr1/Chppr-deficient mice
}

\author{
Massimiliano Monticone ${ }^{1}$, Laura Tonachini ${ }^{1}$, Sara Tavella ${ }^{1}$, Paolo Degan ${ }^{2}$, Roberta Biticchi ${ }^{1}$, \\ Fioretta Palombi ${ }^{3}$, Rossella Puglisi ${ }^{3}$, Carla Boitani ${ }^{3}$, Ranieri Cancedda ${ }^{1,2}$ and Patrizio Castagnola ${ }^{2}$ \\ ${ }^{1}$ DOBIG, Università di Genova, 16131 Genova, Italy, ${ }^{2}$ Istituto Nazionale per la Ricerca sul Cancro, Largo R Benzi \\ 10, 16132 Genova, Italy and ${ }^{3}$ Dipartimento di Istologia ed Embriologia Medica, Università di Roma 'La Sapienza', \\ 00161 Rome, Italy
}

Correspondence should be addressed to P Castagnola; Email: patrizio.castagnola@istge.it

M Monticone and L Tonachini contributed equally to this work

\begin{abstract}
Mtfr1/Chppr is a nuclear gene coding for a mitochondrial protein capable of inducing fission of this organelle in a sequence-specific manner. Here we show that in mice, Mtfr1/Chppr is ubiquitously expressed and displays the highest level of expression in pubertal and adult testes and in particular in spermatids and Leydig cells. To investigate Mtfr1 function in vivo, we analyzed homozygous mice null for this gene obtained through a gene trap approach. We show that these mice fail to express Mtfr1 and that in their testes several genes coding for enzymes involved in the defense against oxidative stress are downregulated. Among these, we studied in particular glutathione peroxidase 3 and show its expression in selected testis cell types. Furthermore, we demonstrate oxidative DNA damage specifically in testes of Mtfr1-deficient mice likely resulting from a reduced antioxidant activity. As a whole, these data suggest that Mtfr1 protects the male gonads against oxidative stress.

Reproduction (2007) 134 483-492
\end{abstract}

\section{Introduction}

A nuclear gene located on mouse chromosome 3 A3 encodes mitochondrial fission regulator 1 (Mtfr1), a mitochondrial protein containing a short polyprolinerich region previously named CHPPR (chondrocyte protein with a polyproline region; Tonachini et al. 2002). This protein is associated with the mitochondrial inner membrane and induces mitochondrial fission, in a sequence-specific manner, when its expression is driven by a cytomegalovirus promoter in several mammalian cell types (Tonachini et al. 2004). We have also shown that this transition from mitochondrial network to spheroid mitochondria is associated with neither loss of the mitochondrial oxidative function nor loss of the protonic gradient (Tonachini et al. 2004). Mitochondria morphology is a highly dynamic parameter resulting from the regulated balance between fission and fusion events (Nunnari et al. 1997, Rizzuto et al. 1998). As several studies have shown that these events play a role in relevant physiological and pathological processes such as cell differentiation, embryo development, apoptosis, and neurodegeneration (Chan 2006), we were prompted to investigate the in vivo function of Mtfr1. In particular, since no data are available in the literature on the function of this gene in mammals, as a previous study was conducted in vitro using the chick Mtfr1 (Tonachini et al. 2004), we chose the mouse as the animal model for this study.

As a first approach to investigate the function of this gene in vivo, we studied the tissue distribution of Mtfr 1 transcripts in wild-type (wt) mice and unexpectedly found the highest level of expression in the testis in a strictly cell-type-dependent pattern. We then studied Mtfr1-deficient mice obtained by a gene trapping strategy allowing random targeting of genes in embryonic stem (ES) cells (Zambrowicz et al. 1998). A gene expression profile approach revealed that in the testes of these mice, several genes coding for reactive oxygen species (ROS) scavenging enzymes are downregulated. As ROS may cause harmful oxidation of proteins, DNA, and lipids, possibly resulting in genotoxic effects and reduced fertility (Liu et al. 2006), we have investigated in detail the expression of several members of the antioxidant system. In 
particular, detailed analysis of glutathione peroxidase 3 (Gpx3) revealed that this enzyme is specifically expressed in the endocrine compartment of the testis and its expression is markedly reduced in Mtfr1deficient mice. Moreover, a survey of different organs for oxidative DNA damage in Mtfr1-deficient mice showed that this specifically affects the testis. The results of this study suggest that Mtfr1 plays a role in the regulation of the expression of ROS scavenging genes in the testis.

\section{Materials and Methods}

\section{Animals}

Wt $\mathrm{C} 57 \mathrm{Bl} / 6 \mathrm{~J}$ mice and Mtfr1-deficient mice were purchased from Charles River (Wilmington, MA, USA) and Lexicon Genetics (The Woodlands, TX, USA) respectively. Mice were bred and maintained at the Institution's animal facility. The care and use of the animals were in compliance with laws of the Italian Ministry of Health and the guidelines of the European Community.

\section{Cell isolation}

Highly purified pachytene spermatocytes and round spermatids (steps 1-8) were obtained from 28- to 30-day-old mouse testes as previously described (Boitani et al. 1980). Briefly, the cell suspension obtained by enzymatic digestion of testicular tissue was fractionated by velocity sedimentation at unit gravity on $0.5-3 \%$ albumin gradient. Purity of cell fractions was verified by flow cytometry and morphology of cytospinned and stained cell preparations (Puglisi et al. 2003). Highly purified type A spermatogonia were obtained from 7-day-old mouse testis as previously described (Morena et al. 1996). Briefly, the cell suspension obtained following enzymatic digestion of testicular tissue was plated for $1 \mathrm{~h}$ on plastic dishes coated with Datura stramonium agglutinin (Sigma). Cells non-adhering to the lectin were fractionated on a discontinuous percoll density gradient (Pharmacia Biotech), giving a cell fraction containing at least $85 \%$ type A spermatogonia.

Sertoli cells were isolated from 14-day-old mice as described by Schlatt et al. (1996) and exposed to hypotonic treatment to eliminate contaminating germ cells (Galdieri \& Zani 1981). In our hands, Sertoli cell cultures appeared to contain $<10 \%$ myoid cells as determined by alkaline phosphatase staining.

Isolated interstitial cells were prepared from 60-day-old mouse testes by collagenase/dispase treatment as described by Mather et al. (1981). In this interstitial cell preparation, Leydig cells were the most represented cell type $(40-45 \%)$ as assessed by histochemical staining for $3 \beta$-hydroxysteroid deydrogenase (Payne et al. 1980). Isolated seminiferous tubules from 60-day-old mouse testes were sedimented after collagenase/dispase digestion and washed twice with PBS.

\section{RNA isolation, CDNA synthesis and Semiquantitative RT-PCR}

Total RNA was extracted from testes of mice at different ages or from isolated testicular cell populations, using the guanidinium thiocyanate-cesium chloride ultracentrifugation method (Chirgwin et al. 1979).

Total RNA from mouse tissues was extracted using TRIzol reagent (Invitrogen) according to manufacturer's protocol. To synthesize the first-strand cDNA, 1-5 $\mu \mathrm{g}$ total RNA were used in a $20 \mu \mathrm{l}$ reaction with the oligo $(\mathrm{dT})_{12-18}$ primer and the superscript first-strand synthesis system for RT-PCR (Invitrogen). Semi-quantitative PCR using the housekeeping gene glyceraldehyde-3-phosphate dehydrogenase (Gapdh) as internal standard was employed to analyze gene expression in mouse tissues. The intensity of the Gapdh amplicon was used to ensure that equal quantities of cDNA from different samples were used in PCR amplification. The optimal primer pair for each gene candidate was designed by the GeneWorks software (IntelliGenetics Inc., Cambell, CA, USA). Primers, probes, and cDNA sequence accession numbers for each gene assayed are listed in Table 1. The amplicon sizes ranged from 190 to $600 \mathrm{bp}$. The PCR program consisted of one cycle at $95^{\circ} \mathrm{C}$ for $1 \mathrm{~min}$ $30 \mathrm{~s}$, then 28-40 cycles (the number of PCR cycles and the annealing temperature were optimized in each case to ensure that intensity of each amplicon fell within the linear phase of amplification) at $95{ }^{\circ} \mathrm{C}$ for $30 \mathrm{~s}$ for denaturing, $58{ }^{\circ} \mathrm{C}-60^{\circ} \mathrm{C}$ for $30 \mathrm{~s}$ for annealing, and $72{ }^{\circ} \mathrm{C}$ for $45 \mathrm{~s}$ for extension. PCR products were separated on 1.5-2\% agarose gel, documented by Gel Doc 1000 (Bio-Rad), and quantified by Molecular Analyst software (Bio-Rad).

\section{Northern blot analysis}

Total RNAs were electrophoresed through 1\% agarose gels in the presence of formaldehyde and blotted onto Hybond $\mathrm{N}$ membranes (Amersham). Blot pre-hybridizations were performed at $65^{\circ} \mathrm{C}$ for $30 \mathrm{~min}$ in $333 \mathrm{mM} \quad \mathrm{NaH}_{2} \mathrm{PO}_{4} \quad \mathrm{pH} \quad 7.2, \quad 6.66 \% \quad \mathrm{SDS}$ and $250 \mu \mathrm{g} / \mathrm{ml}$ denatured salmon sperm DNA. Blot hybridization was performed at $65{ }^{\circ} \mathrm{C}$ for $18 \mathrm{~h}$ in the same solution containing $10^{6} \mathrm{cpm} / \mathrm{ml}$ of denatured and labeled Mtfr1 or Gpx3 coding sequence (cds) probes. After hybridization, the blots were washed twice at $65{ }^{\circ} \mathrm{C}$ for $15 \mathrm{~min}$ in $0.2 \% \mathrm{SDS}, 2 \times \mathrm{SSPE}$, and twice at $65^{\circ} \mathrm{C}$ for $15 \mathrm{~min}$ in $0.2 \%$ SDS, $0.2 \times$ SSPE. Digital 


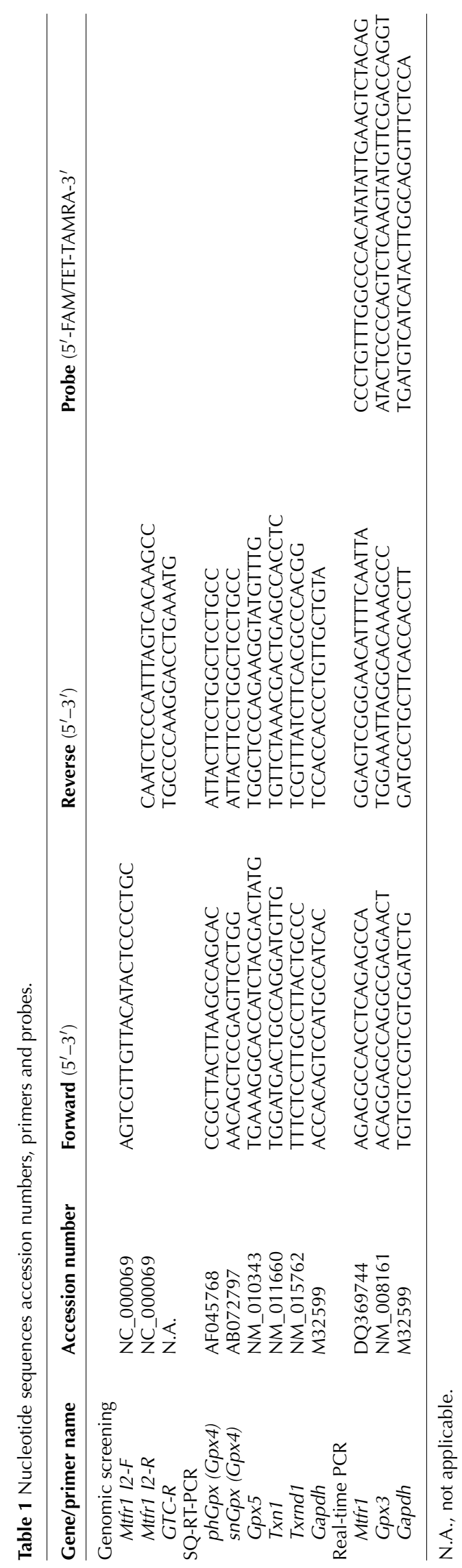

images of blots were acquired using the Cyclone phosphor imaging system (Packard Instruments, Meridien, CT, USA).

\section{Real-time quantitative RT-PCR}

Relative quantification was performed with the $A B I$ Prism 7700 Sequence Detector (Applied Biosystems, Foster City, CA, USA) following manufacturer's instructions. The housekeeping gene Gapdh was used as the endogenous control for normalization. To avoid possible signal production from potential contaminating genomic DNA, specific primers and probes for each gene were designed across a common exon-intron splice junction by the Primer Express software (Applied Biosystems). Primers, probes, and cDNA sequence accession numbers for each gene assayed are listed in Table 1.

\section{Generation and characterization of Mtfr1-deficient mice}

The mutation in the mouse genomic DNA was generated by the gene trap method (Zambrowicz et al. 1998). The gene trap vector was used to generate a random mutant library (Omnibank Library, Lexicon Genetics) of ES cells derived from the 129/SvEvBrd mouse. In the library, we identified the clone OST6261 in which Mtfr1 was disrupted. By using this clone, the C57BL/6J heterozygous mice were generated by Lexicon Genetics and crossed to obtain homozygous mutant mice. The mutation was confirmed by Southern blot and PCR analysis using genomic DNAs isolated from the mouse tail. For Southern blotting, genomic DNA was isolated from the mouse tail using the Puregene kit (Gentra Systems Inc., Minneapolis, MN, USA) and digested with Nhel (Roche Applied Science). Ten microgram cleaved DNA fragments were separated by $0.75 \%$ agarose gel and blotted after denaturation, and neutralization onto Hybond $\mathrm{N}$ membranes following the manufacturer's instructions (GE Healthcare Europe Gmbh, Milan, Italy). The blots were hybridized with a probe for Mtfr1 exons 2-3 generated by PCR using mouse Mtfr 1 cDNA (Accession number DQ369744) with primers 5'-CTGCTATGGATGCCTGAAGT-3' and 5'-ACTGAATCAGAGATAAGTTGGTACC-3'. Southern blot pre-hybridization, hybridization, and washing were performed at $68{ }^{\circ} \mathrm{C}$ using solutions of the same composition indicated for northern blot analysis. About $10^{6} \mathrm{cpm} / \mathrm{ml}$ denatured $\left[{ }^{32} \mathrm{P}\right]$-labeled DNA fragment spanning nucleotides 200-400 of the mouse Mtfr1 sequence with Accession Number DQ369744 was used as probe. Digital images of blot were acquired using a cyclone phosphor imaging system (Packard Instruments). 


\section{Reproductive performance test}

To assess reproductive performance,couples of 6-monthold male and female mice were kept in the same cage, until appearance of a vaginal plug, for 5 days a week for a total of 4 weeks. Litter size was recorded within $24 \mathrm{~h}$ of delivery.

\section{Histology, histochemistry, and electron microscopy}

Samples for conventional histology were stained in carmalum after fixation in Bouin. For histochemical detection of the $\beta$ galactosidase:neomycin phosphotransferase chimera $(\beta G E O)$ expression, tissue samples were fixed for $30 \mathrm{~min}$ in $0.2 \%$ formaldehyde, $5 \mathrm{mM}$ EGTA, and $2 \mathrm{mM} \mathrm{MgCl} 2$ in PBS. The tissues were washed (thrice, $30 \mathrm{~min}$ each) in $0.1 \mathrm{M}$ phosphate buffer $\mathrm{pH} 7.3$ containing $2 \mathrm{mM} \mathrm{MgCl} 2,0.01 \%$ sodium deoxycholate, and $0.02 \%$ NP40, then incubated overnight in a 5-bromo-4-chloro-3indolyl- $\beta$-D-galactopyranoside (X-Gal) staining solution (2 $\mathrm{mM} \mathrm{MgCl}, 0.01 \%$ sodium deoxycholate, $0.02 \%$ NP40, $5 \mathrm{mM}$ potassium hexacyanoferrate (II) and (III), $1 \mathrm{mg} \mathrm{X}$-Gal per $\mathrm{ml}$, in $0.1 \mathrm{M}$ phosphate buffer $\mathrm{pH} 7.3$ ). After staining, samples were washed with PBS, dehydrated in graded ethanol and embedded in paraffin. Sections $(10 \mu \mathrm{m})$ were cut and routine observations and image acquisition were performed with a Zeiss Axiovert 200 M microscope (Carl Zeiss, Jena, Germany). Samples for ultrastructural observation were fixed in $2.5 \%$ buffered glutaraldehyde and postfixed in 1\% osmium tetroxide, then conventionally dehydrated in ethanol and embedded in Epon. Semithin sections stained in buffered toluidine blue were analyzed to select the precise step of spermiogenesis to investigate at the ultrastructural level. Ultrathin sections were stained in uranyl acetate and lead citrate and were studied and photographed in a Hitachi 7000 transmission electron microscope (Hitachi).

\section{Mitochondrial labeling and epifluorescence imaging}

Fibroblasts were collected by enzymatic dissociation performed as previously described (Castagnola et al. 1986) from 12 days post coitum (p.c.) mouse embryos. The cells, from wt and homozygous Mtfr1-deficient embryos were plated on glass coverslips and cultured in DMEM (Sigma) supplemented with 10\% fetal bovine serum and $5 \mathrm{mM}$ glutamine (Invitrogen). To visualize mitochondria and nuclei, the coverslips were incubated in culture medium containing $23.4 \mathrm{nM}$ Mitotracker Red CMXRos (Invitrogen) and $4 \mu \mathrm{g} / \mathrm{ml}$ Hoechst 33432 (Invitrogen) for $20 \mathrm{~min}$ at $37^{\circ} \mathrm{C}$. After two brief washes, the cells were incubated again for additional $20 \mathrm{~min}$ at $37^{\circ} \mathrm{C}$ with culture medium and immediately fixed at $22{ }^{\circ} \mathrm{C}$ for 5 min with $3.7 \%$ paraformaldehyde in PBS with $2 \%$ sucrose. After fixation, the coverslips were briefly washed first in PBS and then in distilled water and finally mounted on glass slides for microscopy examination. Optical sections $(500 \mathrm{~nm})$ were generated by means of structured illumination using an Axiovert $200 \mathrm{M}$ microscope equipped with Apotome and driven by the Axiovision software (Carl Zeiss).

\section{Hybridization of cDNA Arrays}

For hybridization of Atlas Plastic Mouse 5K microarrays (Takara Bio Europe/Clontech), $\left[{ }^{33} \mathrm{P}\right]$-labeled probes were generated by RT of $50 \mu \mathrm{g}$ total RNA according to the manufacturer's instructions. After hybridization, filters were scanned using the Cyclone phosphor imaging system (Packard Instruments). Data were processed using Clontech Atlaslmage 2.01 software. Intensities were adjusted through a median normalization and differential expression was arbitrarily considered significant when signal ratio for the same spot was $\geq 1.9$.

\section{Evaluation of oxidative damage}

DNA was extracted from different mouse tissues by using Puregene reagent (Gentra) according to the manufacturer's protocol. The analysis of 8-hydroxy-2'-deoxyguanosine $(8-\mathrm{OHdG})$ content in DNA was performed by high performance liquid chromatography with electrochemical detection (HPLC/EC) as described previously (Degan et al. 1995) using genomic DNA from mouse tissues. The levels of 8 -OHdG were expressed as the number of $8-\mathrm{OHdG}$ adducts per $10^{6}$ deoxyguanosine bases in the samples.

\section{Statistical analysis}

The data were examined using unpaired Student's t-test. The results were considered to be statistically significant when $P<0.05$.

\section{Results}

\section{Mtfr1 is ubiquitously expressed in mouse tissues with} the highest expression found in the testis

To assess the expression of Mtfr1 in wt adult mouse tissues, we performed a Northern blot analysis. This showed that Mtfr1 is expressed in all wt tissues to varying degrees with the highest level found in the testis (Fig. 1A). In particular, all the tissues examined displayed a single Mtfr1 mRNA of about $2.8 \mathrm{~kb}$, whereas in testis we detected two additional mRNA species of about 2.4 and $1.4 \mathrm{~kb}$ respectively (Fig. $1 \mathrm{~A}$ ). To determine the coding sequence (cds) of this $1.4 \mathrm{~kb}$ transcript, we performed a cDNA cloning from a testis cDNA library. We isolated a cDNA clone that confirmed the presence of an Mtfr1-specific cDNA of size corresponding to the 

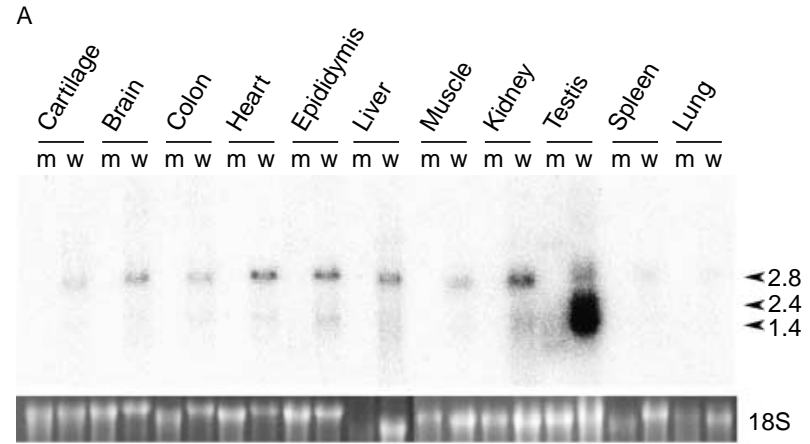

B

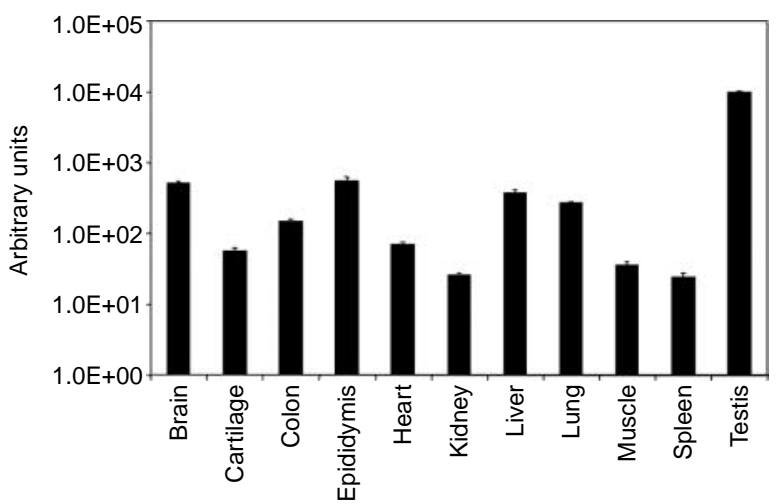

Figure 1 Mtfr1 expression in mouse tissues. (A) Northern analysis of Mtfr1 transcripts in mouse tissues. RNA was extracted from tissues of 6-month-old wild-type (wt) and homozygous Mtfr1-deficient mice (hom) for the trapped allele. Arrowheads indicate mRNA length in kb. At the bottom, the $18 \mathrm{~S}$ rRNA region of the gel is shown as loading control. (B) Real-time RT-PCR analysis showing relative Mtfr1 transcripts levels in RNA extracted from tissues of a 6-month-old wild-type mouse, log scale. Expression levels, in arbitrary units, are relative to the expression of Gapdh; the s.D. is indicated.

lowest mRNA species detected by northern blot analysis (GB Accession DQ369744, data not shown). Furthermore, the analysis of the nucleotide sequence of this cDNA along with the analysis of the sequence of other cDNA sequences obtained from testis available in the GenBank demonstrate that the corresponding transcripts are alternatively spliced in this tissue in order to yield Mtfr 1 mRNAs with different $5^{\prime}$ or $3^{\prime}$ UTRs but with a conserved coding region when compared with the single Mtfr 1 mRNA obtained from other tissues (data not shown). To establish relative expression levels of Mtfr1 mRNAs in wt mouse tissues, we performed a real-time RT-PCR analysis. By this assay, we observed that Mtfr1 expression in testes is one or two logs higher with respect to other tissues (Fig. 1B).

\section{Gene targeting effectively inactivates the Mtfr1 locus}

To gain more insight into the in vivo function of Mtfr1, mice deficient for the expression of this gene were obtained from Lexicon Genetics. These mice resulted from a gene trap procedure performed through introduction in ES cells of the retroviral vector VICTR 21 and subsequent generation of chimeric blastocysts (Zambrowicz et al. 1998). We confirmed by nucleotide sequencing, Southern blot, and PCR analyses that these mice harbor a copy of the vector inserted in the intron located between exons 2 and 3 of the Mtfr1 gene (Fig. 2A-C). In the present report, we show data obtained studying mice homozygous for the Mtfr1 trapped allele referred to as Mtfr1-deficient or Mtfr1-mutant mice. As demonstrated by Northern blot analysis, the expression of $M t f r 1$ is undetectable in Mtfr1-deficient mice (Fig. 1A). As expected in embryonic and adult tissues of these mice, we detected the expression of the $\beta$ GEO marker, encoded by the gene trap vector, and driven by the promoter of the trapped Mtfr1 gene (Fig. 2A). In particular, the marker staining detected in developing retina, cartilage, perichondrium, and adult testis is shown in Fig. 3.

\section{Mtfr1-deficient mice are viable and fertile}

Mtfr1-deficient mice did not show obvious defects in phenotype and behavior. Histological analysis of their testes displayed morphology similar to that of wt animals (Fig. 3G and H). In addition, mutant mice were found to generate a normal number of pups when compared with wt mice of the same genetic background (C57BL/6J) matched for age (data not shown). The observed frequency of pups bearing the trapped Mtfr1 allele in heterozygosis or homozygosis, born after crossing homozygous mice with heterozygous or wt mice was never significantly different from the expected Mendelian ratio (data not shown). To rule out any reduced reproductive performance of $M t f r 1$-deficient males, we performed a series of breeding experiments and found that males $(n=6)$ homozygous for the trapped Mtfr1 allele, when crossed with wt females, on average generated fewer pups than wt males $(n=8)$ but with such a degree of variability that this difference did not attain statistical significance $(P=0.421)$.

\section{Mitochondria of Mtfr1-deficient mice are morphologically similar to those of wt cells}

Given the ability of Mtfr1 to induce mitochondrial fission in transfected cells (Tonachini et al. 2004), we decided to verify whether its absence resulted in altered mitochondrial morphology. To address this topic, we derived mouse embryo fibroblasts from both wt and Mtfr1deficient mice, cultured them in vitro and stained the mitochondria with the specific fluorescent probe Mitotracker Red. This experiment showed that there do not appear to be gross differences in mitochondrial morphology of Mtfr1-deficient cells with respect to that 


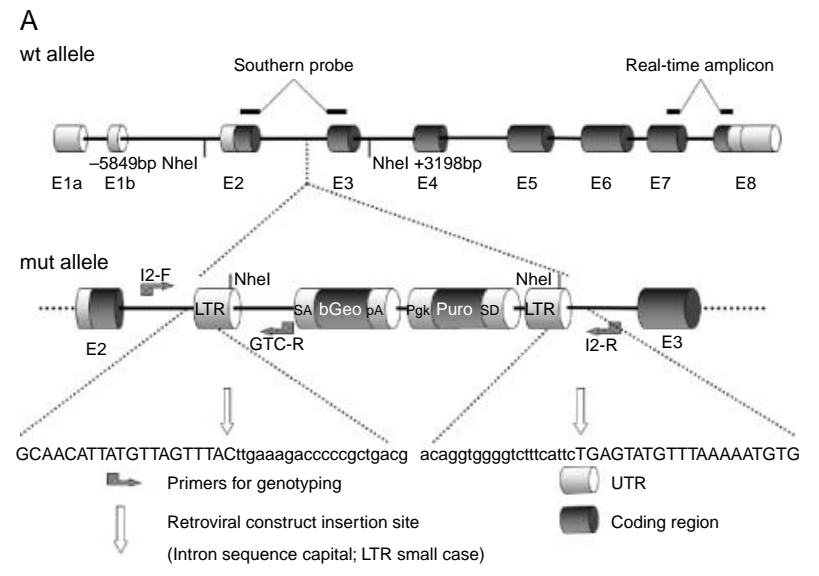

B

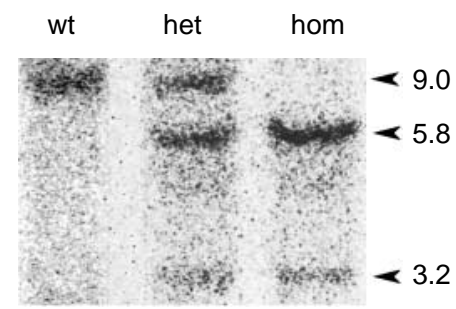

C

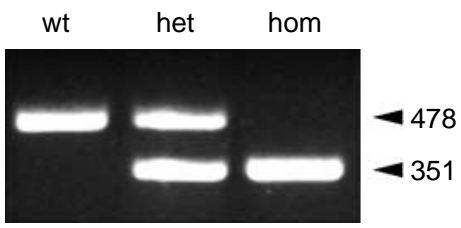

Figure 2 Generation of Mtfr1-deficient mice by gene trapping. (A) Schematic diagram of the gene trap vector (VICTR21) site of insertion into Mtfr1. The restriction sites for Nhel in the vector LTRs and in the wt allele are indicated along with the distance in basepairs relative to the vector insertion site. Arrows indicate the orientation and approximate location of PCR primers listed in Table 1. The wild-type (wt) and the trapped allele (mut) are shown along with the sequence of the novel junction between the vector LTRs (lower case) and the intron located between exons 2 and 3 (upper case). Regions corresponding to sequences used to generate a DNA probe for Southern blot analysis and the amplicon for real-time PCR analysis are indicated. (B) Southern blot analysis with the probe shown in Fig. 2A of genomic DNA digested with Nhel extracted from tails of wt, heterozygote (het), and homozygous (hom) mice for the Mtfr1-trapped allele. Arrowheads indicate DNA fragment size expressed in kbp. (C) PCR analysis of genomic DNA extracted from tails of wt, heterozygote (het), and homozygous (hom) mice for the trapped allele performed with primers I2-F I2-R and GTC-R (indicated in Fig. 2A and Table 1). The PCR conditions used do not allow amplification of the gene-trapped allele with the I2-F I2-R couple of primers. Arrowheads indicate DNA fragment size expressed in bp.

of wt cells (Fig. 4). Furthermore, epifluorescence analysis of spermatozoa labeled with Mitotracker Orange and ultrastructural analysis of spermatids and testicular spermatozoa resulted in similar observations (data not shown).
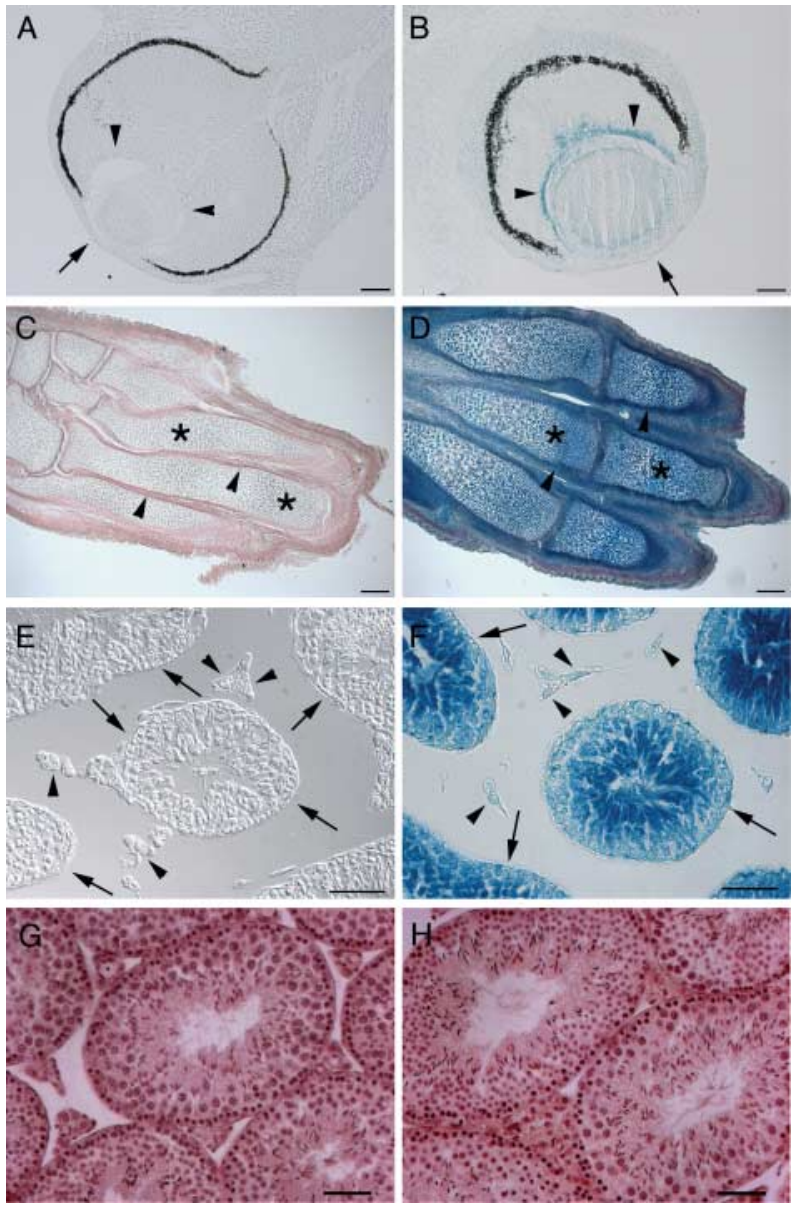

Figure $3 \mathrm{Mtfr} 1$ expression in tissues of Mtfr1-deficient mice. Mtfr1 expression was revealed by $\beta G E O$ expression resulting from the splicing with Mtfr1 exon 2 (Fig. 2A). Wild-type tissues (A, C, E and G); tissues of Mtfr1-deficient mice homozygous for the trapped allele (B, D, $\mathrm{F}$ and $\mathrm{H})$. Eyes and hind digits at $12.5 \mathrm{dpc}(\mathrm{A}$ and $\mathrm{B})$ and $(\mathrm{C}$ and $\mathrm{D})$ respectively. Testes of 6 -month-old mice ( $E$ and $F$ ). ( $A$ and $B$ ) Arrows point to the eye anterior segment whereas arrowheads point to the retinal stratum opticum. (C and D) Arrowheads point to the perichondrium and asterisks indicate developing cartilage.

( $E$ and $F$ ) Arrows indicate seminiferous tubules; arrowheads indicate Leydig cells. ( $G$ and $H$ ) Carmalum stained sections of wt $(G)$ and mutant (H) mouse testes. Bar $=50 \mu \mathrm{m}(\mathrm{A}-\mathrm{D})$. Bar $=100 \mu \mathrm{m}(\mathrm{E}-\mathrm{H})$.

\section{Mtfr1-deficiency results in reduced expression of genes coding for enzymes with oxidoreductase activity in the testis}

To gain insight into the possible molecular alterations caused by lack of expression of Mtfr1 in testes, we performed a global gene expression analysis by cDNA array hybridization, using RNA samples extracted from a pool of mutated and wt testes obtained from several animals $(n=7)$ in order to compensate for individual variation in gene expression. About 5000 genes were assayed, several of which were found to be differentially regulated in testes of $M t f r 1$-deficient mice with respect to 

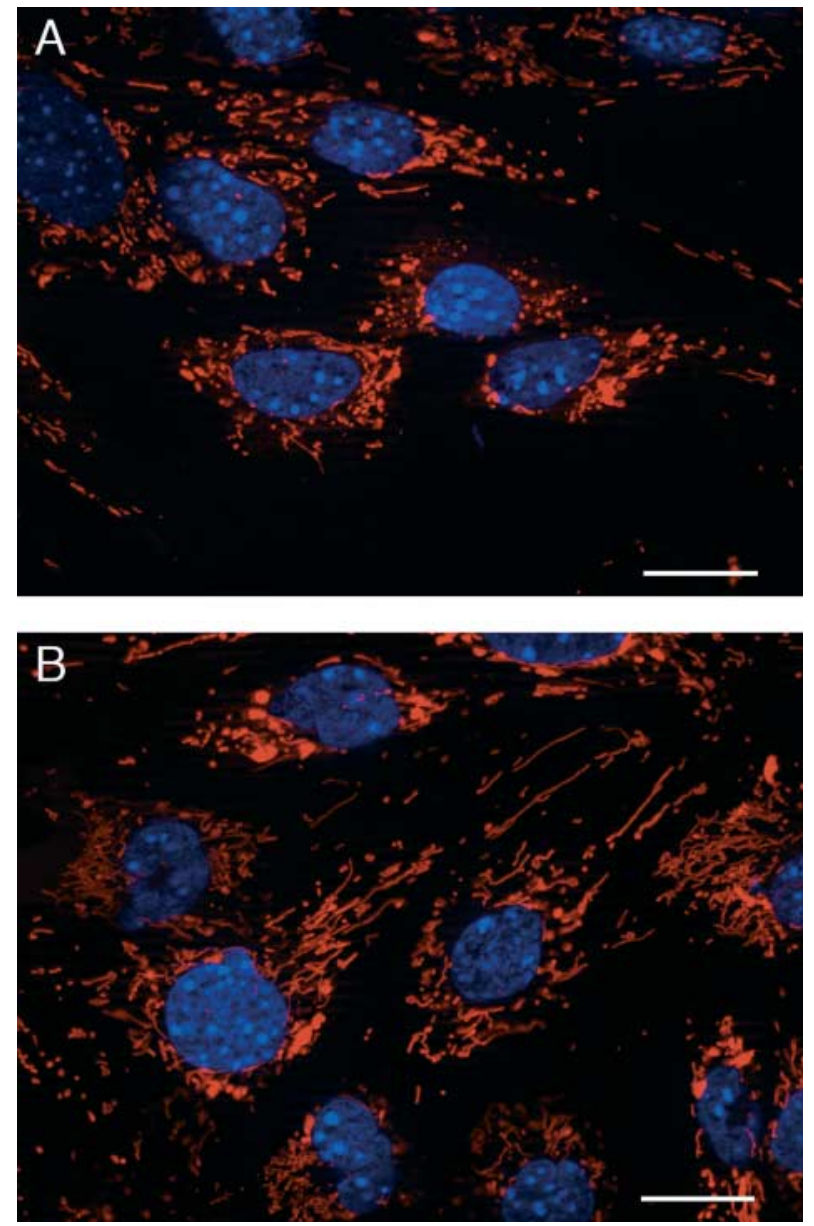

Figure 4 Analysis of mitochondrial morphology. Mouse embryo fibroblast from wt (A) and Mtfr1-deficient mice homozygous for the trapped allele (B) were labeled with Mitotracker Red and Hoechst 33432 to visualize mitochondria and nuclei respectively. Optical sections of $500 \mathrm{~nm}$ generated by means of structured illumination are shown. $\operatorname{Bar}=20 \mu \mathrm{m}$.

wt individuals (data not shown). Among these genes, we focused our attention on the most represented group, which was related to the oxireductive metabolism since mitochondria play a key role in the generation of ROS (Table 2). In order to confirm the regulation of each single gene included in this group, independent semiquantitative or real-time RT-PCR analyses were performed on the same RNA pool used for the cDNA array hybridization. All the genes whose deregulation was confirmed by these experiments were found to be downregulated in the mutant mice to varying extents; these genes were the following: thioredoxin 1 (Txn1), thioredoxin reductase $1(\operatorname{Tn} r d \times 1)$, glutathione peroxidase 4 (Gpx4, coding for mitochondrial and nuclear isoforms of phospholipid hydroperoxide glutathione peroxidase (PHGPX)), Gpx5 and Gpx3 (Fig. 5A and B). We focused our subsequent analyses on Gpx3 since in cDNA array analysis it appeared as the most highly expressed gene among this group (Table 2) and because its expression in
Table 2 Differentially expressed genes, involved in oxidative metabolism, in Mtfr1-deficient mice (mut) with respect to wt mice as identified by DNA array analysis.

\begin{tabular}{|c|c|c|c|c|}
\hline Gene & $\begin{array}{c}\text { Accession } \\
\text { number }\end{array}$ & $\begin{array}{c}\text { Wt/ } \\
\text { Mut } \\
(*)\end{array}$ & $\begin{array}{c}\text { Mut O.D. } \\
\text { arbitrary } \\
\text { units }\end{array}$ & $\begin{array}{l}\text { Wt O.D. } \\
\text { arbitrary } \\
\text { units }\end{array}$ \\
\hline $\begin{array}{l}\text { Androgen } \\
\text { regulated vas } \\
\text { deferens } \\
\text { protein } \\
(\text { Akr1b7) }\end{array}$ & NM_009731 & 7.4 & 42 & 309 \\
\hline $\begin{array}{l}\text { Antioxidant } \\
\text { protein } 1 \\
(\text { Atx } 1 / \text { Atox } 1)\end{array}$ & NM_009720 & 2.2 & 81 & 176 \\
\hline $\begin{array}{l}\text { Cytochrome } c \\
\quad \text { oxidase, } \\
\text { subunit VIIc } \\
(\operatorname{Cox} 7 c)\end{array}$ & NM_007749 & 3.2 & 128 & 413 \\
\hline $\begin{array}{l}\text { Glutathione } \\
\text { peroxidase } 3 \\
\text { (Gpx3) }\end{array}$ & U13705 & 2.1 & 1830 & 3857 \\
\hline $\begin{array}{l}\text { Glutathione } \\
\text { peroxidase } 4 \\
(G p \times 4)\end{array}$ & NM_008162 & 2.1 & 598 & 1232 \\
\hline $\begin{array}{l}\text { Glutathione } \\
\text { peroxidase } 5 \\
(G p \times 5)\end{array}$ & NM_010343 & 1.9 & 96 & 182 \\
\hline $\begin{array}{l}\text { Thioredoxin } 1 \\
\text { (Txn1) }\end{array}$ & NM_011660 & 2.0 & 151 & 299 \\
\hline $\begin{array}{l}\text { Thioredoxin } \\
\text { reductase } 1 \\
(\operatorname{Tnrd} 1 \times 1)\end{array}$ & NM_015762 & 2.1 & 97 & 203 \\
\hline
\end{tabular}

$\left({ }^{*}\right) \mathrm{Wt} /$ Mut represents the ratio of gene expression in wild-type animals versus Mtfr1-deficient mice. This ratio has been calculated from the optical density O.D., expressed in arbitrary units, resulting from the DNA array hybridization analysis. The values relative to the most expressed gene, Gpx3, are shown in bold.

the male gonad has been poorly characterized. To investigate the differential expression of Gpx3 mRNA at an individual level among wt and Mtfr1-deficient mice, a second group of animals were killed and Gpx3 mRNA levels measured in the testes of each mouse. This analysis demonstrated that individual variations in Gpx3 expression within both wt and Mtfr1-deficient animals occur and, although the differential expression observed between wt and Mtfr1-deficient testes is less prominent with respect to the pooled samples, the reduced Gpx3 mRNA expression in Mtfr1-deficient mice remains statistically significant $(P<0.01$; Fig. 5C).

\section{Mtfr1 deficiency leads to oxidative DNA damage in the testis}

The downregulation in Mtfr1-deficient mice testes of several genes coding for enzymes with antioxidant activity prompted us to investigate the presence of oxidative damage in the tissues of these mice. To this end, we evaluated by HPLC/EC the $8 \mathrm{OHdG} / 2$ '-deoxyguanosine $(\mathrm{dG})$ ratio, a marker of oxidative DNA damage (Shigenaga et al. 1989). The amount of $8 \mathrm{OHdG}$ was found to be about two times higher in the homozygous 
Mtfr1-deficient than in the wt testes $(n=3 ; P=0.0007)$ whereas in other tissues such as liver and lung, no significant differences were detected with respect to the wt mice (Fig. 6).

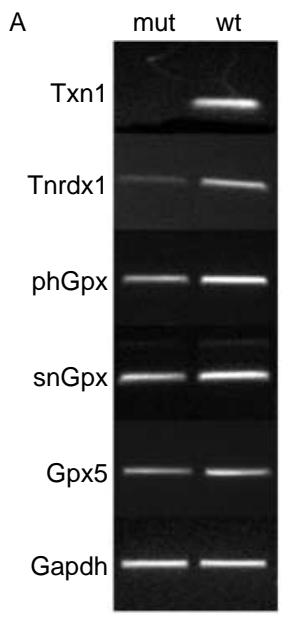

B

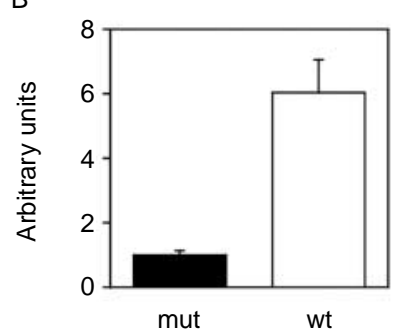

C

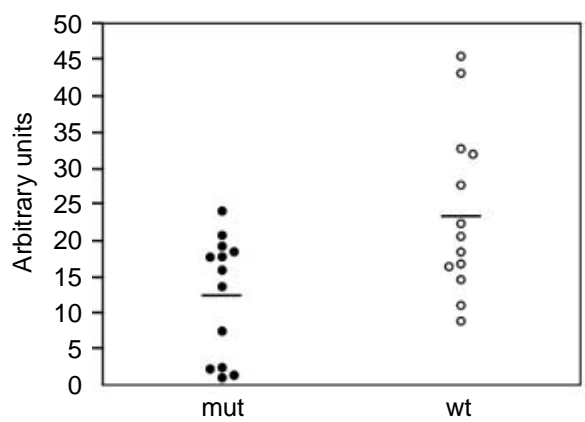

Figure 5 Analysis of expression levels of genes coding for ROS scavenger enzymes in Mtfr1-deficient mice. (A) Semiquantitative RT-PCR analysis performed with the primers indicated in Table 1 specific for the indicated genes. RNA was extracted from a pool of $(n=7)$ testes of 6-month-old wild-type (wt) or Mtfr1-deficient mice homozygous for the trapped allele (mut). (B) Real-time RT-PCR analysis showing Gpx3 transcripts levels. RNA was extracted from a pool of $(n=7)$ testes of 6-month-old wild-type (wt, white bar) or homozygous Mtfr1-deficient mice for the trapped allele (mut, black bar). Expression levels, in arbitrary units, are relative to the expression of Gapdh; the SD is indicated. (C) Real-time RT-PCR analysis showing relative Gpx3 mRNA levels in individual testes collected from $(n=13)$ Mtfr 1 -deficient mice homozygous for the trapped allele (black dots) or from $(n=13)$ wild-type mice (white dots). Expression levels, in arbitrary units, are relative to the expression of Gapdh; black lines represent mean values for each group, $P<0.01$.

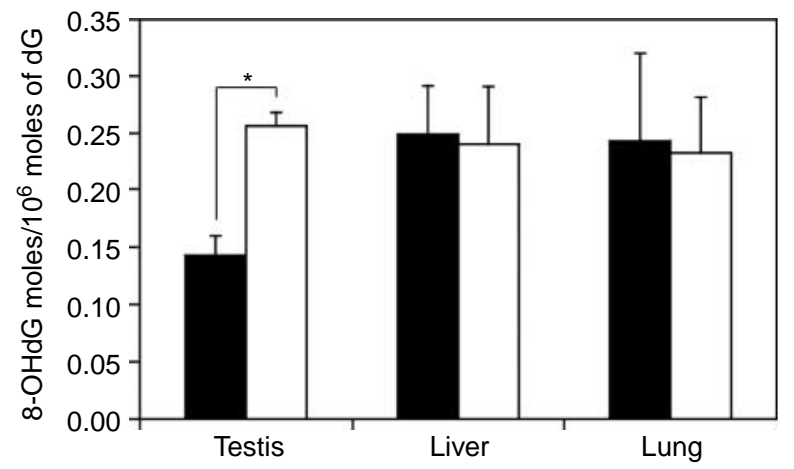

Figure 6 Oxidative damage in testes of Mtfr 1 -deficient mice. Relative levels of $8-\mathrm{OHdG}$ in genomic DNA extracted from tissues of 6-monthold Mtfr1-deficient mice homozygous for the trapped allele (white bars) or from wild-type mice (black bars). The data are expressed as the ratio of $8-\mathrm{OHdG}$ moles $/ 10^{6}$ moles of dG measured by HPLC/EC. A representative experiment done using three DNA samples for each group is shown; each of the DNA samples was measured in triplicate; the SD is indicated. Asterisk indicates $P=0.0007$.

\section{Selected cell types in the testis express Mtfr1 and Gpx3}

To establish the timing of Mtfr1 and Gpx3 expression during testis maturation, and which testicular cell types express these genes, we performed Northern blot analyses on immature and adult samples. In the total testis, the $2.8 \mathrm{~kb} M t f r 1$ ubiquitous transcript was barely detectable (Fig. 7), whereas the expression of two shorter transcripts of 2.4 and $1.4 \mathrm{~kb}$ was apparent, the latter being more abundant. Interestingly, the presence of the two testis-specific transcripts was observed starting from 30 days after birth, when a large number of haploid cells
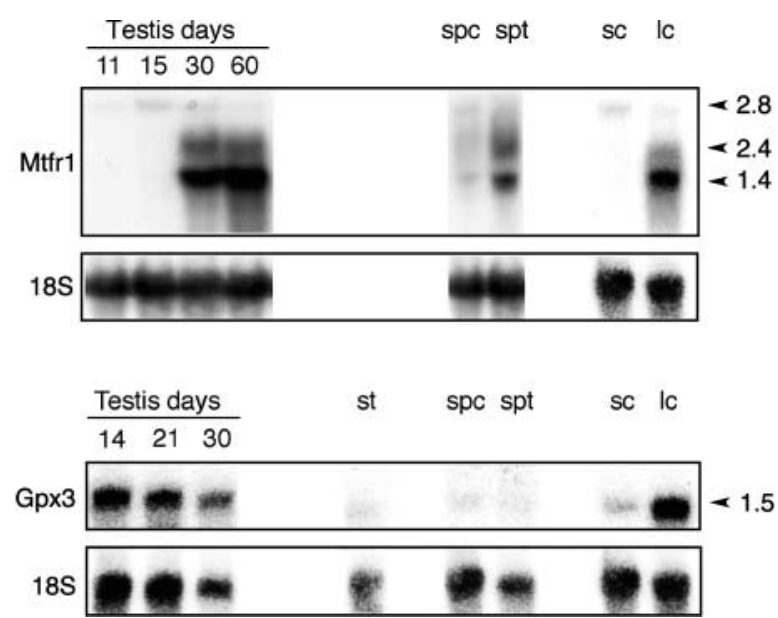

Figure 7 Expression of $M$ tfr 1 and Gpx3 in mouse total testis and different testicular cell types. Wt mouse testes were collected at the indicated days after birth whereas seminiferous tubules (st) were collected at 60 days. Spc, Spt, SC, and LC indicate middle-late pachytene spermatocytes, round spermatids, Sertoli cells, and Leydig cells respectively. Arrowheads indicate mRNA length in $\mathrm{kb}$. At the bottom of each panel, the $18 \mathrm{~S}$ rRNA region of the gel is shown as loading control. 
first appear in seminiferous tubules, and maintained thereafter (Fig. 7). When we analyzed selected testis cell types by northern blotting, the two short Mtfr1 transcripts were found at low level in middle-late pachytene spermatocytes and at higher level in round spermatids and in a sample of interstitial tissue enriched in Leydig cells (Fig. 7). Sertoli cells (Fig. 7) and spermatogonia (not shown) did not express detectable levels of these two Mtfr 1 testis-specific mRNAs.

The analysis of Gpx3 expression in the testis demonstrated that a single $1.5 \mathrm{~kb}$ mRNA is expressed in early pubertal gonad and maintained thereafter with no major quantitative changes. As for Gpx3 expression in testicular cell types, the transcript was absent in both meiotic and post-meiotic cells, barely detectable in Sertoli cells, and particularly abundant in an interstitial cell sample enriched in Leydig cells (Fig. 7). In line with this localization in the seminiferous tubules from adult testes Gpx3 mRNA was barely detectable (Fig. 7).

\section{Discussion}

Our results concerning the ubiquitous expression of this gene in mouse tissues are in agreement with data available in the public domain (http://www.genecards. org/cgi-bin/carddisp.pl?gene=MTFR1; Rebhan et al. 1997). However, here we report for the first time that in mouse tissues Mtfr 1 is expressed at the highest level in pubertal and adult testes, in particular by the haploid germ cells and Leydig cells. In the testis, we show that the Mtfr1 primary transcript is processed to generate specific Mtfr1 mRNAs sharing the same cds with the ubiquitous Mtfr 1 mRNA but differing in their $5^{\prime}$ and $3^{\prime}$ UTRs.

Mice homozygous for the presence of a gene trap vector located between exons 2 and 3 of the Mtfr 1 gene did not express $M t f r 1$ transcripts in any tissue examined by Northern analysis, and are therefore to be considered as Mtfr1 deficient.

Our results indicate that although expression of Mtfr1 by transfection in vitro induces mitochondrial fission in several cell types (Tonachini et al. 2004), Mtfr1 loss does not overtly affect mitochondrial morphology. This evidence strengthens our previous hypothesis that Mtfr1 acts as a regulator/facilitator of the fission process triggered by other factors. Interestingly, our finding that Mtfr1 is expressed in round spermatids is consistent with the observation that the recognized key regulators of the mitochondrial fission and fusion processes, dynaminrelated protein 1 and mitofusin 2, are expressed at the highest level in pubertal and adult rat testis in the same germ cell type (Honda \& Hirose 2003). Therefore, it is possible that Mtfr1 participates to the unusual mitochondrial dynamics observed during spermatogenesis (Honda \& Hirose 2003, McQuibban et al. 2006), although our ultrastructural analysis did not detect any apparent alteration in the homozygous mouse testis. The observation of a late Mtfr1 expression during male germ cells differentiation suggests a possible function of the Mtfr1 protein in spermatozoa.

Unexpectedly, our study shows that in the testes of Mtfr1-deficient mice, the expression of several genes involved in the defense against oxidative stress is abnormal. In particular, we found a decreased expression of Gpx3,Txn1,Tnrdx1 and, at a lesser extent, of Gpx4 and Gpx5. These enzymes are scavengers of ROS acting as a coordinate network against oxidative damage (Mates et al. 1999, Finkel 2003, Vernet et al. 2004, Hanukoglu 2006). We also demonstrate the expression of Gpx3 in interstitial Leydig cells, to our knowledge a novel finding, which may open new investigation perspectives on the antioxidant role of this enzyme in this endocrine tissue. Here, we show that the reduced expression of antioxidant genes in Mtfr1deficient testes is accompanied by oxidative DNA damage. Interestingly, a similar condition has been reported in aging skin (Lu et al. 1999, Wei \& Lee 2002). However, despite this pro-oxidant phenotype induced in their testes, Mtfr1-deficient males displayed no apparent fertility impairment, which might possibly require the occurrence of a pathological oxidative stress. To this respect, it should also be taken into account that in the testis, a major contribution in ROS scavenging is deployed by Gpx4 (Imai et al. 2001, Puglisi et al. 2005), the expression of which appears only slightly inhibited by Mtfr1 loss.

We speculate that the impact of Mtfr1 loss on both the mitochondrial morphology and the pro-oxidant status might be attenuated by the expression of the Mtfr1 paralog, which could provide a redundant function. This is a poorly studied gene named Dufd 1 located on chromosome $10 \mathrm{~A} 3$ encoding a putative protein sharing a $34 \%$ identity with the Mtfr1 protein (data not shown).

Experimental evidence provided here indicates that Mtfr 1 expression is prominent in testes and suggests that this gene is involved in the regulation of antioxidant activity in the male gonad. However, it remains to be established whether this function and the ability to induce mitochondrial fission observed elsewhere (Tonachini et al. 2004) are related to each other, and how Mtfr1 influences the expression of genes coding for ROS scavenger enzymes. We forward the hypothesis that this influence is indirect, likely exerted upon Leydig cells (in which both Mtfr1 and Gpx3 are expressed) and possibly resulting from effects this mitochondrial protein might exert on the metabolism of the mitochondrion itself.

\section{Acknowledgements}

We thank Raffaela Arbicò for expert technical assistance and Marina Fabbi for helpful discussion and suggestions. Research supported by The European Union (STREP PIONEER 
FOOD-2005-513991) to C B. The authors declare that there is no conflict of interest that would prejudice the impartiality of this scientific work.

\section{References}

Boitani C, Geremia R, Rossi R \& Monesi V 1980 Electrophoretic pattern of polypeptide synthesis in spermatocytes and spermatids of the mouse. Cell Differ 9 41-49.

Castagnola P, Moro G, Descalzi-Cancedda F \& Cancedda R 1986 Type $\mathrm{X}$ collagen synthesis during in vitro development of chick embryo tibial chondrocytes. Journal of Cell Biology 102 2310-2317.

Chan DC 2006 Mitochondria: dynamic organelles in disease, aging, and development. Cell 125 1241-1252.

Chirgwin JM, Przybyla AE, MacDonald RJ \& Rutter WJ 1979 Isolation of biologically active ribonucleic acid from sources enriched in ribonuclease. Biochemistry 18 5294-5299.

Degan P, Bonassi S, De Caterina M, Korkina LG, Pinto L, Scopacasa F, Zatterale A, Calzone R \& Pagano G 1995 In vivo accumulation of 8-hydroxy-2'-deoxyguanosine in DNA correlates with release of reactive oxygen species in Fanconi's anaemia families. Carcinogenesis 16 735-741.

Finkel T 2003 Oxidant signals and oxidative stress. Current Opinion in Cell Biology 15 247-254.

Galdieri M \& Zani B 1981 Hormonal induced changes in sertoli cell glycoproteins. Cell Biology International Reports $\mathbf{5} 111$.

Hanukoglu I 2006 Antioxidant protective mechanisms against reactive oxygen species (ROS) generated by mitochondrial P450 systems in steroidogenic cells. Drug Metabolism Reviews 38 171-196.

Honda S \& Hirose S 2003 Stage-specific enhanced expression of mitochondrial fusion and fission factors during spermatogenesis in rat testis. Biochemical and Biophysical Research Communications 311 424-432.

Imai H, Suzuki K, Ishizaka K, Ichinose S, Oshima H, Okayasu I, Emoto K, Umeda M \& Nakagawa Y 2001 Failure of the expression of phospholipid hydroperoxide glutathione peroxidase in the spermatozoa of human infertile males. Biology of Reproduction 64 674-683.

Liu Z, Lin H, Ye S, Liu Q-y, Meng Z, Zhang C-m, Xia Y, Margoliash E, Rao Z \& Liu X-j 2006 Remarkably high activities of testicular cytochrome $\mathrm{c}$ in destroying reactive oxygen species and in triggering apoptosis. PNAS 103 8965-8970.

Lu CY, Lee HC, Fahn HJ \& Wei YH 1999 Oxidative damage elicited by imbalance of free radical scavenging enzymes is associated with large-scale mtDNA deletions in aging human skin. Mutation Research 423 11-21.

Mates JM, Perez-Gomez C \& Nunez de Castro I 1999 Antioxidant enzymes and human diseases. Clinical Biochemistry 32 595-603.

Mather JP, Saez JM \& Haour F 1981 Primary cultures of Leydig cells from rat, mouse and pig: advantages of porcine cells for the study of gonadotropin regulation of Leydig cell function. Steroids 38 35-44.

McQuibban GA, Lee JR, Zheng L, Juusola M \& Freeman M 2006 Normal mitochondrial dynamics requires rhomboid-7 and affects Drosophila lifespan and neuronal function. Current Biology $\mathbf{1 6}$ 982-989.
Morena AR, Boitani C, Pesce M, De Felici M \& Stefanini M 1996 Isolation of highly purified type A spermatogonia from prepubertal rat testis. Journal of Andrology 17 708-717.

Nunnari J, Marshall WF, Straight A, Murray A, Sedat JW \& Walter P 1997 Mitochondrial transmission during mating in Saccharomyces cerevisiae is determined by mitochondrial fusion and fission and the intramitochondrial segregation of mitochondrial DNA. Molecular Biology of the Cell 8 1233-1242.

Payne AH, Downing JR \& Wong KL 1980 Luteinizing hormone receptors and testosterone synthesis in two distinct populations of Leydig cells. Endocrinology 106 1424-1429.

Puglisi R, Tramer F, Panfili E, Micali F, Sandri G \& Boitani C 2003 Differential splicing of the phospholipid hydroperoxide glutathione peroxidase gene in diploid and haploid male germ cells in the rat. Biology of Reproduction 68 405-411.

Puglisi R, Tramer F, Carlomagno G, Gandini L, Panfili E, Stefanini M, Lenzi A, Mangia F \& Boitani C 2005 PHGPx in spermatogenesis: how many functions? Contraception 72 291-293.

Rebhan M, Chalifa-Caspi V, Prilusky J \& Lancet D 1997 GeneCards: integrating information about genes, proteins and diseases. Trends in Genetics 13163.

Rizzuto R, Pinton P, Carrington W, Fay FS, Fogarty KE, Lifshitz LM, Tuft RA \& Pozzan T 1998 Close contacts with the endoplasmic reticulum as determinants of mitochondrial $\mathrm{Ca} 2+$ responses. Science 280 1763-1766.

Schlatt S, de Kretser DM \& Loveland KL 1996 Discriminative analysis of rat Sertoli and peritubular cells and their proliferation in vitro: evidence for follicle-stimulating hormone-mediated contact inhibition of Sertoli cell mitosis. Biology of Reproduction 55 227-235.

Shigenaga MK, Gimeno CJ \& Ames BN 1989 Urinary 8-hydroxy-2'deoxyguanosine as a biological marker of in vivo oxidative DNA damage. PNAS 86 9697-9701.

Tonachini L, Monticone M, Di Marco E, Zerega B, Cancedda R \& Castagnola P 2002 Chondrocyte protein with a poly-proline region is a novel protein expressed by chondrocytes in vitro and in vivo. Biochimica et Biophysica Acta 1577 421-429.

Tonachini L, Monticone M, Puri C, Tacchetti C, Pinton P, Rizzuto R, Cancedda R, Tavella S \& Castagnola P 2004 Chondrocyte protein with a poly-proline region (CHPPR) is a novel mitochondrial protein and promotes mitochondrial fission. Journal of Cellular Physiology $201470-482$.

Vernet P, Aitken RJ \& Drevet JR 2004 Antioxidant strategies in the epididymis. Molecular and Cellular Endocrinology 216 31-39.

Wei YH \& Lee HC 2002 Oxidative stress, mitochondrial DNA mutation, and impairment of antioxidant enzymes in aging. Experimental Biology and Medicine 227 671-682.

Zambrowicz BP, Friedrich GA, Buxton EC, Lilleberg SL, Person C \& Sands AT 1998 Disruption and sequence identification of 2,000 genes in mouse embryonic stem cells. Nature 392 608-611.

Received 2 May 2007

First decision 1 June 2007

Accepted 19 June 2007 